\title{
Parton Distributions at the LHeC
}

\author{
Max Klein \\ University of Liverpool, Department of Physics \\ L69 7ZE Liverpool, United Kingdom
}

\begin{abstract}
The LHeC provides $e^{ \pm} p$ collisions at $1.4 \mathrm{TeV}$ cms energy. Based on simulations of the statistics and the systematics as may be achieved with a new collider detector, the potential of the $\mathrm{LHeC}$ is characterised regarding DIS precision measurements as of the strong coupling constant, to per mille accuracy, and the gluon distribution. It is demonstrated that for the first time the flavour contents of the proton can be completely reconstructed. Precision measurements will be possible of the up, down, strange, charm, beauty, anti-up, anti-down and anti-strange quark distributions over a huge range in $x$ and $Q^{2}$ hitherto not accessible. This is solely based on high precision neutral and charged current measurements, in $e p$ and partially in $e D$ scattering, using charm and beauty tagging for the reconstruction of the heavy quark momentum distributions.
\end{abstract}

\section{Introduction}

The Large Hadron Electron Collider (LHeC) project envisages deep inelastic electron and positron proton collisions using a new electron ring mounted on top of the LHC. With an electron energy $E_{e}$ of typically $70 \mathrm{GeV}$ in collisions with the LHC proton beam of $E_{p}=$ $7 \mathrm{TeV}$, the LHeC achieves electron-quark collisions at a center of mass energy of up to $\sqrt{s}=4 E_{e} E_{p}=1.4 \mathrm{TeV}$ at which new physics may be found, as the formation of resonant $e q$ states. The design, as proposed in [1], promises to achieve luminosities of $10^{33} \mathrm{~cm}^{-2} \mathrm{~s}^{-1}$, using the standard LHC proton beam, or possibly higher if the luminosity upgrade of the LHC was realised.

A $\mathrm{TeV}$ energy scale $e p$ collider is of primary importance to study new physics in the $e q$ sector, to study the origin of mass, as is linked to the strong self-interaction of gluons, the confinement of quarks and deconfinement phase of partonic matter in nuclei, to move the deep investigation of proton structure much further, as with searches for substructure, study of parton correlations or transverse structure, to measure parton distributions in nuclei, understand the formation of the quark gluon plasma and to explore the strong interaction dynamics much deeper. In the present note it is demonstrated, based on a full simulation of systematic detector uncertainties, that the $\mathrm{LHeC}$ for the first time will enable the complete decomposition of proton structure into its partonic base ${ }^{\mathrm{a}}$. Such a decomposition is achieved with the precise measurement of all partonic parameters, $\alpha_{s}, x g, u_{s}, u_{v}, \bar{u}, d_{s}, d_{v}, \bar{d}, s, \bar{s}$, $c, \bar{c}$ and of $b, \bar{b}$, using inclusive neutral (NC) and charged current (CC) simulated data only. Low $x$ physics and physics beyond the Standard Model with the LHeC were discussed at this workshop in $[2,3]$.

\footnotetext{
${ }^{\text {a }}$ Measurements of parton distributions in nuclei so far exist only from fixed target $l N$ scattering experiments. The LHeC by using the LHC ion beams will extend these by four orders of magnitude in $x$ and $Q^{2}$ and thus provide a basis for understanding phenomena as predicted in the theory of the colour-glass condensate and for interpreting data which will be taken by ALICE and the other LHC collider experiments in $A A$ mode. Most striking phenomena are predicted in high energy $e A$ scattering such as an increase of the relative amount of diffraction up to $50 \%$ or scaling laws of $F_{2}$ much different from Bjorken's predictions or logarithmic $Q^{2}$ variations in high energy ep scattering.
} 
The precision measurement of parton distribution functions (pdf) in the kinematic range of the LHC, as will be provided by the LHeC, will essentially remove the otherwise neccessary assumptions on evolving measurements from the restricted HERA range into the kinematic region of the LHC. While one may hope to be able to control such an evolution in $Q^{2}$ at medium $x$, HERA simply has not the coverage of the small $x$ region in which new saturation phenomena may occur. Measurements at high masses at the LHC may require a very accurate knowledge of the valence quark densities at $Q^{2}$ of order $10^{5 . .6} \mathrm{GeV}^{2}$. It is to be noted, however, that HERA was seriously luminosity limited to reach high $x>0.5$. With LHC $p p$ data some of the evolutions from HERA using perturbative QCD may be tested, as from the Drell Yan production of the weak bosons. However, these processes are also considered to allow for a precision determination of the luminosity of the LHC, and apart from a few exceptions as the $W^{ \pm}$asymmetry one hardly can determine the luminosity and simultaneously the pdf's in the kinematic range of the LHC, see also [4].

\section{Simulation and Detector Requirements}

To achieve maximum luminosity, for $Q^{2}$ above $100 \mathrm{GeV}^{2}$, the $e p$ interaction region at the LHeC, as considered in [1], has focusing quadrupoles placed near the vertex. This limits the forward and backward acceptance to polar angles between $10^{\circ}$ and $170^{\circ}$. The region close to the beam pipe, however, is crucial for the physics at low $x$ and for measuring the final state at large $x$ and medium $Q^{2} \sim 10^{4 . .5} \mathrm{GeV}^{2}$. It may be accessed with removal of these magnets at an estimated tolerable reduction of the luminosity by a factor of about 10 . In this study it is assumed that tracking and calorimetry may then be extended to $1^{\circ}$ and $179^{\circ}$. Thus there are two basic challenges for the LHeC detector(s) and interaction region: the accurate reconstruction of scattered electron and final state energies up to $\sim 5 \mathrm{TeV}$ and the forward backward instrumentation close to the beam pipe to reliably access lowest and large $x$. The third challenge is to achieve maximum precision. This is quantified here by considering the relation of calibration and general measurement accuracy to the precision with which the strong coupling constant $\alpha_{s}\left(M_{Z}^{2}\right)$ can be measured. In an initial case study, here a first set of detector requirements was considered, see Table 1, roughly two times more ambitious than H1, 30 years later in modern technology developments.

\begin{tabular}{|l|l|l|}
\hline Parameter & H1 & LHeC \\
\hline Polar angle acceptance [ ${ }^{\circ}$ ] & $7-177$ & $1-179$ \\
Polar angle measurement accuracy [mrad] & $0.2-1$ & $0.1-0.5$ \\
Hadronic energy scale [\%] & 2 & 1 \\
Electromagnetic energy scale [\%] & $0.2-1$ & 0.1 \\
Luminosity measurement accuracy [\%] & 1 & 0.5 \\
\hline
\end{tabular}

Table 1: Acceptance and measurement accuracies typical for the H1 detector at HERA and assumed in this study for the $\mathrm{LHeC}$ detector. The scale uncertainties at the LHeC are chosen to achieve better than $1 \%$ cross section measurement accuracy in most of the kinematic region. They roughly match the statistical uncertainty at large $x$ for $Q^{2}$ above $10000 \mathrm{GeV}^{2}$ for $10 \mathrm{fb}^{-1}$. Further studies will define this more accurately and less globally.

Simulations of NC and CC inclusive cross sections were performed for data sets of $10 \mathrm{fb}^{-1}$ at high $Q^{2}$ and $1 \mathrm{fb}^{-1}$ at low $Q^{2}$, for polar angles inside $10-170^{\circ}$ and $1-179^{\circ}$, respec- 
tively. The systematic errors were derived using electroweak Born cross section formulae from the dependence of the kinematic variables on the electron and hadron final state energies and angles with the uncertainties as given in Table 1 . The numeric calculation of systematic uncertainties has been cross checked with Monte Carlo simulations for HERA. The $\Sigma$ method was assumed for reconstruction and a global uncertainty of $0.5 \%$ was added to the systematic errors.

\section{Strong Coupling Constant and Gluon Distribution}

The strong coupling $\alpha_{s}$ is known to at best $1 \%$ accuracy, worse even than the gravitational constant. The present uncertainty of $\alpha_{s}$ limits the evaluation of grand unification of the weak, electromagnetic and strong interactions at the Planck scale. DIS is known to be a process, calculated so far to NNLO, which is best suited for a measurement of $\alpha_{s}\left(M_{Z}^{2}\right)$. Using NLO QCD fit programs the estimated experimental uncertainty of $\alpha_{s}\left(M_{Z}^{2}\right)$ is \pm 0.0004 for the $\mathrm{LHeC} e^{ \pm} p$ data and \pm 0.0003 when the simulated LHeC data are combined with the BCDMS $\mu p$ data. Further studies are foreseen to evaluate deeper the effects of individual uncertainties and acceptance constraints in order to understand the requirements to the LHeC detector in more detail, attempting to come close to a per mille measurement accuracy. Yet, the present result already indicates the exciting potential precision DIS measurements at the $\mathrm{LHeC}$ have. It also poses a considerable challenge to QCD calculations since at NNLO the conventional measurement uncertainty due to the arbitrariness of the renormalisation scale is quoted to be about \pm 0.001 related to the HERA range.

The gluon distribution from the present DIS data, even including di-jet cross sections, is rather inaccurately determined, in particular at low $x$ and at large $x$. This is a major concern for the LHC as $g g$ fusion processes are often the dominant production mechanism. An analysis similar to the one for $\alpha_{s}\left(M_{Z}^{2}\right)$ reveals that the wide range and high precision of $\mathrm{LHeC}$ data would allow the gluon distribution to be determined much more accurately than has been possible so far, both at low and at high $x$, see Figure 1.

\section{Quark Distributions}

With the LHeC, the light quark densities $u, \bar{u}, d$ and $\bar{d}$ can be determined with very high accuracy over the full range of $x$ from NC, CC, ep and $e d$ data. This will resolve the long standing question on the behaviour of $u / d$ at large $x$, see Figure 1 , and determine the $d\left(x, Q^{2}\right)$ density to high accuracy. From the electroweak beam charge asymmetry a determination is obtained of high accuracy of the valence quarks, $x F_{3}^{\gamma Z} \propto 2 u_{v}+d_{v}$, down to $x=10^{-3}$, i.e. in a region one never could probe the valence quarks in DIS so far.

HERA has measured the density of beauty quarks in the proton for the first time, not far from threshold and to a limited accuracy of $20 \%$ for $Q^{2}$ up to a few $100 \mathrm{GeV}^{2}$. It has provided more accurate measurements of the charm quark density to 5-10\%. HERA has not measured the strange quark distribution.

Assuming $50 \% b$ impact parameter tagging efficiency, $\epsilon_{b}$, and a charm background fraction, $b g_{c}$, of $10 \%$, a very accurate measurement of $b\left(x, Q^{2}\right)$ can be done, for $x \simeq 0.00003-0.07$ and $Q^{2}$ from threshold to $10^{5} \mathrm{GeV}^{2}$. In the same range $c\left(x, Q^{2}\right)$ can be measured well, as is obtained with $\epsilon_{c}=0.1$ and a light quark background $b g_{q}=0.01$. Using the charged current reaction $W^{+} s \rightarrow c$ and its charge conjugate, for the first time $s\left(x, Q^{2}\right)$ and $\bar{s}\left(x, Q^{2}\right)$ become measurable, for $x$ between about 0.001 and 0.1 and $Q^{2}$ between 500 and $50000 \mathrm{GeV}^{2}$. Such 

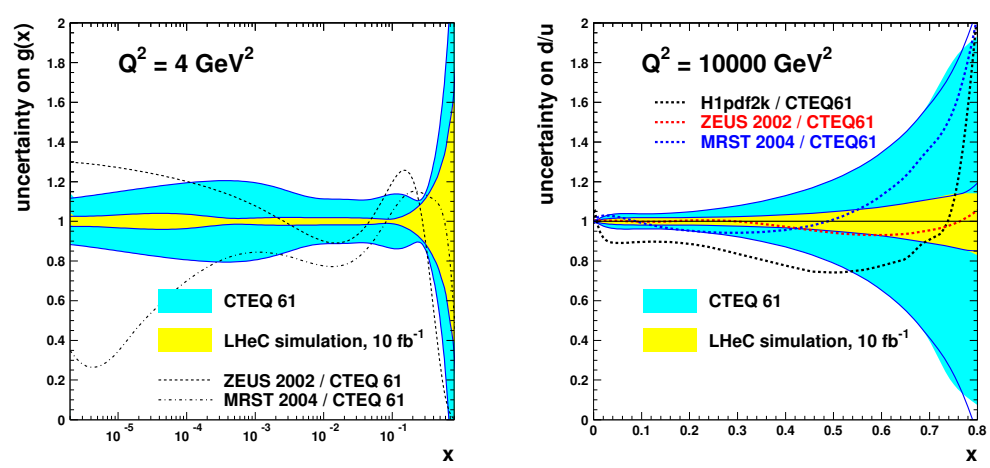

Figure 1: The gluon distribution (left) and the $u / d$ ratio (right) as a function of $\log x$ and of $x$, respectively. The blue (outer) band illustrates the uncertainty of the CTEQ6.1 pdf set, the yellow (inner) band is the experimental uncertainty as determined in this analysis. The curves show by how much current QCD fits may differ, often outside the quoted uncertainty from a particular set. The present knowledge of $x g$ is expected to be constrained better from the forthcoming final HERA data on the $\ln Q^{2}$ derivative of $F_{2}$ and on $F_{L}$.

measurements, simulated here with impact parameter evaluations only, will profit further from dedicated Silicon vertex detectors, extending to the forward region, and from the small beam spot size at the $\mathrm{LHeC}$ of $35 \times 15 \mu \mathrm{m}^{2}$ only.

The measurement of parton distributions with the LHeC represents the culmination of decades of pdf measurements in lepton-nucleon DIS experiments and may turn out to be of crucial relevance for the interpretation of new physics phenomena one expects to find both in $p p$ and also in $A A$ collisions at the LHC. For the slides and plots of this talk see [5].

\section{Acknowledgements}

Thanks are due to T. Kluge and E. Perez for performing QCD fits on the simulated data to obtain $\alpha_{s}$ and pdf's, respectively, further to A. Mehta for guidance on the heavy quark density measurement simulation and my coauthors of [1] for much excitement and insight. Thanks to the organizers for a great workshop at Munich.

\section{References}

[1] J. Dainton et al., JINST 1 P10001 (2006) [hep-ex/0306016]; For a recent review see J. Dainton, these Proceedings.

[2] P. Newman, these Proceedings.

[3] E. Perez, these Proceedings.

[4] M. Cooper-Sarkar, these Proceedings.

[5] Slides: http://indico. cern. ch/contributionDisplay py? contribId=255\&sessionId=10\&conf Id=9499 